Los gobiernos locales y su rol estratégico en la integración regional: una apuesta hacia la integración

subnacional en el MERCOSUR

DOI: https://doi.org/10.24215/24689912e030

\title{
LOS GOBIERNOS LOCALES Y SU ROL ESTRATÉGICO EN LA INTEGRACIÓN REGIONAL: UNA APUESTA HACIA LA INTEGRACIÓN SUBNACIONAL EN EL MERCOSUR
}

\section{LOCAL GOVERNMENTS AND THEIR STRATEGIC ROLE IN REGIONAL INTEGRATION: A COMMITMENT TO SUBNATIONAL INTEGRATION IN THE MERCOSUR}

Nahuel Avaredo

Marcelo Arzamendia

\begin{abstract}
RESUMEN
El presente trabajo expone el incipiente proceso de internacionalización de los gobiernos locales y su influencia en la construcción de los procesos de integración regional. La integración a nivel subnacional puede consolidar y complementar un proceso mayor como es la integración regional del MERCOSUR. Se analizan los factores más relevantes que han posibilitado la acción internacional y paradiplomática de los gobiernos no centrales y, particularmente, de los gobiernos locales como son las redes de ciudades, la cooperación transfronteriza y otras plataformas de interacción horizontal. El papel protagónico que hoy asumen los gobiernos locales en la profundización de los procesos de integración regional permitiría avanzar hacia una más inclusiva, en consonancia a la integración supranacional de los Estados, y asimismo ampliar la agenda y la toma de decisiones desde la perspectiva local, muchas veces postergada en el bloque sudamericano.
\end{abstract}

\section{PALABRAS CLAVE}

Integración regional, Integración subnacional, Gobernanza multinivel, Paradiplomacia, Gobiernos locales

\section{ABSTRACT}

The present work exposes the incipient process of internationalization of local governments and its influence in the construction of regional integration processes. Integration at the sub-national level can consolidate and complement a larger process such as MERCOSUR's regional integration. The most relevant factors that have made possible the international and paradiplomatic action of the non-central governments and, particularly, of the local governments, such as the networks of cities, the cross-border cooperation and other platforms of horizontal interaction, are analyzed. The leading role assumed today by local governments in the deepening of regional integration processes would make it possible to advance towards a more inclusive integration, in line with the supranational integration of States, and also to expand the agenda and decision-making from the local perspective, often neglected in the South American bloc.

\section{KEY WORDS}

Regional integration, Subnational integration, Multi-level governance, Paradiplomacy, Local governments 


\section{INTRODUCCIÓN}

El creciente protagonismo que han tomado los gobiernos no centrales durante las últimas décadas en el amplio espectro de las relaciones internacionales los posiciona indefectiblemente a ser considerados como focos de estudios dentro de la academia. Los Estados subsisten y se transforman constantemente y paralelamente los gobiernos no centrales han mutado en el alcance de sus competencias y sus capacidades, separándose incluso de la concepción clásica del realismo que admite la actuación soberana y casi exclusiva de los Estados nacionales en el desarrollo del orden internacional, concibiendo una nueva perspectiva. Este fenómeno conlleva transformaciones que han permitido nuevas alternativas e interacciones en el esquema tradicional $y$, en el caso de los gobiernos locales, la aparición de instituciones que definen sus propias políticas, estrategias y acciones de cooperación internacional. Los gobiernos locales y los intermedios (provincias, estados, departamentos y otras denominaciones) configuran los gobiernos no centrales, pero son los primeros los que presentan unas dinámicas en constante crecimiento que demandan un mayor estudio. De este modo, la descentralización es un factor gravitante al momento de analizar la vinculación extraterritorial de los gobiernos locales, una manifestación originaria desde las antiguas civilizaciones.

Paradójicamente, en medio de diversas dificultades y limitaciones, los gobiernos locales han luchado intensamente por instalar su propia agenda en el sistema internacional a través de diversos mecanismos de gobernanza multinivel o experiencias paradiplomáticas. Lograron la creación de significativas redes de interacción y cooperación descentralizada, cuyo punto de inflexión se indexa en las numerosas demandas a favor de sus habitantes, asiduamente caracterizada por aquellas necesidades de primer orden, ya sea salud, educación, vivienda, alimentación, movilidad, acceso a energía eléctrica, agua potable y otros bienes y servicios públicos esenciales, en torno al desarrollo social, económico y por sobre todo sustentable y sostenible de la comunidad. El rol de los gobiernos locales ya no se extiende dentro de los límites de sus fronteras, ya que constantemente se encuentran en la necesidad de gestionar escenarios inciertos y vulnerables que emergen del propio contexto internacional. En este sentido, Marx (2010) plantea que el Estado ha diseminado ciertas capacidades a los estratos de orden local y regional, permitiendo la transferencia de ciertos tipos de poderes políticos, económicos, 
administrativos y fiscales, en la búsqueda de soluciones ante las demandas sociales que se caractericen por la solidaridad y cooperación.

Por consiguiente, los gobiernos locales hoy desempeñan un rol protagónico en la consolidación de los procesos de integración regional (PIR) llevados adelante in extenso a nivel mundial -Unión Europea (UE), Mercado Común del Sur (MERCOSUR), Comunidad Andina, entre otros-. En estos bloques regionales tienen lugar el desarrollo y la inserción de actores subnacionales, robusteciendo un modelo de integración denominada integración subnacional. Esta interacción entre los PIR y la integración subnacional genera una relación binaria e interdependiente y de constante retroalimentación, positiva desde donde se la observe y, principalmente, a favor de la construcción de una identidad o ciudadanía regional, con avances justos y equilibrados, muchas veces relegada por el mainstream tradicional de la integración regional, que no se caracteriza por priorizar la inclusión y las demandas de la población.

Consecuentemente, el MERCOSUR a lo largo de sus treinta años no ha abandonado la etapa estatista, en comparación a la UE, que sí ha avanzado en el montaje de políticas y marcos institucionales, concediendo espacios de desenvolvimiento a los gobiernos locales $\mathrm{y}$, de este modo, reforzando la creación de una verdadera ciudadanía regional. No obstante, estas unidades subnacionales continúan pugnando por su participación en la toma de decisiones, indispensable ante el contexto del mundo de hoy, que incita a remarcar la resiliencia de estos actores y a una constante reinvención.

El objetivo de este artículo es destacar el papel estratégico de los gobiernos locales en la consolidación de los PIR, abordando el caso de la integración subnacional en el MERCOSUR, en base a la configuración recíproca de lo subnacional a lo regional, aggiornada a una compatibilidad dinámica y mutua, a través de su participación multinivel en redes de cooperación que se ajusta cada vez más al diseño de una integración subnacional. Se valida la construcción de este trabajo mediante la revisión bibliográfica de enfoques teóricos con respecto a la temática propuesta. En la primera sección, se delimita una perspectiva teórica acerca de la internacionalización de los gobiernos locales; de la misma forma, se realiza una aproximación en relación a los PIR y su vinculación a dichas unidades 
subnacionales. En la segunda sección, se plantea la paradiplomacia y la integración regional como procesos paralelos e intrínsecos, además de conceptualizar la integración subnacional y su manifestación en el MERCOSUR a través de Mercociudades y distintas instancias de cooperación transfronteriza. Finalmente, el trabajo concluye con una reflexión sobre el tópico desarrollado.

\section{Perspectiva teóricas}

\subsection{El surgimiento de nuevos actores en las relaciones internacionales}

La globalización ha puesto en un gran dilema al paradigma estatocéntrico, especialmente al momento de focalizar los retos y desafíos disruptivos que se concibieron en el sistema internacional durante las últimas décadas en asuntos políticos, sociales, culturales y económicos. Keohane y Nye (1988) dilucidaron bajo la teoría de la interdependencia compleja que los gobiernos centrales ya no son los únicos entes exclusivos que confluyen en las relaciones transnacionales, sino que se encuentran acompañados de actores no gubernamentales, movimientos sociales, asociaciones y diversas organizaciones de carácter transgubernamental, contrastando con la concepción realista. En este contexto, los autores destacan la desaparición de la línea divisoria entre política exterior y política doméstica, pero aclaran que la desaparición de estos límites no representa un obstáculo en un mundo cada vez más interdependiente.

Por su parte, Del Arenal (2002) sostiene que la heterogeneidad y complejidad del sistema internacional posee relación directa en la pluralidad de actores, en primer lugar por el debilitamiento del rol central del Estado, que ha cedido espacios frente a otros actores dejando de ser el único actor del sistema, y, en segundo lugar, por la aparición en la escena internacional de actores de distinta naturaleza, cuyo crecimiento y preponderancia se relacionan con las dinámicas de la interdependencia, la globalización y la transnacionalización.

Esta nueva configuración provocó un cambio de rumbo de las relaciones internacionales: la agenda internacional se ha extendido a varios actores, ya no precisamente de índole estatal, y las relaciones transgubernamentales fueron prosperando en el ámbito de las unidades subnacionales, como es el caso de los gobiernos locales. Rosenau (1990) agrega que los actores subnacionales serían los 
propulsores de las relaciones transgubernamentales, ubicándose en lo que él domina el mundo multicéntrico de las relaciones internacionales, evolucionando en nuevos actores internacionales con el deseo de proyectar sus intereses. Asimismo, el costo y la oportunidad de la internacionalización varía por sector; en este sentido, las ventajas comparativas de las unidades subnacionales se fortalecen a través de las redes de interacción local e internacional. Esta idea se enlaza con lo descrito por Keohane y Nye (1971) acerca de los grupos internos que desarrollan el encono suficiente como para activar una cuestión que dormía o su capacidad de interferir en los más altos niveles de negociación interestatal.

Ahora bien, la internacionalización de las unidades subnacionales es un proceso que comprende la creación de capacidades autónomas con proyecciones de inserción al orden internacional; de este modo, el papel exclusivo y casi hegemónico de los Estados en la diplomacia tradicional se ha diluido en demasía (Botto, 2013). Es así que nuevos competidores surgen, en efecto, ante un hecho sin precedentes como es la ruptura del patrón estatocéntrico, reconfigurando las categorías de alta y baja política y, por ende, la reubicación de la autoridad hacia actores subnacionales y no estatales propiciaron un cúmulo de cambios en el sistema internacional. De otro modo, las unidades subnacionales reciben elementos estratégicos directos e indirectos del sistema internacional, transformando estos instrumentos en sus propios intereses, e incluso trasladándose a otras esferas lejanas de sus fronteras.

La gestión internacional de las unidades subnacionales con respecto a las transformaciones del orden internacional les ha permitido adquirir una mayor preferencia y predominio en la línea transgubernamental (Juste, 2017). En suma, los gobiernos no centrales se han apropiado de una mayor flexibilización e innovación en las relaciones internacionales, a la par de los Estados (Cruz, Manassero y Piana, 2019).

Otra cuestión no menos importante es aquella relacionada a los aspectos legales e institucionales que avalan el desempeño internacional de los actores subnacionales, variando el grado de autonomía y competencias que los Estados otorgan a los gobiernos no centrales (Allas Proyecto, 2013). En el caso de Paraguay, cuya estructura política está descentralizada en gobiernos departamentales y municipales, la Constitución Nacional, en los artículos 167, 168 y 171, expresa las prerrogativas y 
atribuciones que corresponden a los gobiernos locales; asimismo, establece que podrán asociarse entre sí para encarar en común la realización de sus fines y, mediante ley, con municipalidades de otros países. Sin embargo, considerando el artículo 1, 137 y 141 de dicha Carta Magna (Constitución Nacional del Paraguay, 1992), además de la Ley Orgánica Municipal del Paraguay, los acuerdos, tratados y acciones de carácter internacional son atribuciones particulares y exclusivas del gobierno central. Esto difiere en el caso de Argentina o Brasil, que sí disponen de un marco legal que permite a sus gobiernos provinciales y Estados federales una acción más dinámica y descentralizada en el exterior.

\subsection{Los procesos de integración regional, el neofuncionalismo y la gobernanza multinivel}

La situación actual que atraviesan los diversos PIR merece una profunda revisión, y a su vez una reflexión crítica acerca de sus principios, intereses y desafíos, constantemente degradados por las elites gobernantes. Lógicamente, al momento de dirigir los esfuerzos en comprender las particularidades de este proceso, es ideal partir del concepto clásico, acuñado por Haas (1958), que en primera instancia se refirió a la integración regional como "el proceso por el cual diversos actores políticos y de distintos ámbitos estatales aceptan desviar sus lealtades, expectativas y actividades políticas hacia un nuevo centro, cuyas instituciones poseen o demandan una jurisdicción sobre los Estados nacionales preexistentes" (p.16). En los años posteriores, Haas (1970) describió la integración regional como un proceso por el cual los países se mezclan, confunden y fusionan con sus vecinos, de modo que ceden ciertos atributos fácticos de la soberanía a la vez adquieren nuevas herramientas para resolver sus conflictos mutuos.

Por otro lado, la CEPAL (2014) considera que la integración regional es un proceso multidimensional que abarca más allá de los ejes meramente económicos y comerciales, incluyendo además políticas sociales, culturales y ambientales. Malamud (2011) acota además que es una etapa de transición entre una situación conocida y otra en construcción, no obstante, su origen es voluntario y su estadio final es indeterminado; asimismo, el autor sostiene que el proceso como sí nace y es conducido formalmente en el seno de los Estados. 
Consecuentemente, la idea de integración frente al auge de la globalización se forjó muchas veces desde las esferas económicas, con patrones elitistas y sesgados en el campo de políticas de promoción y preferencias comerciales, subyacentes a estrategias neoliberales y de crecimiento económico, que suprimieron tópicos y sectores sociales relevantes ante la falta de interés y dejaron de lado la participación de actores ajenos al gobierno central. Todo ello demostró significativamente la necesidad de ampliar las reformas estructurales en la concepción de un PIR que, en el caso del MERCOSUR, encontró más palos que zanahorias. ${ }^{1}$

Del mismo modo, el desenvolvimiento sui generis de los diversos PIR desarrollados en América Latina y el Caribe evidenciaron vacíos sociales y políticos, cuyo efecto arrojó a mediano plazo la incapacidad de llevar adelante un verdadero proyecto de integración y cooperación, profundo y transversal, en sintonía a la construcción de una agenda inclusiva que contrasta la actuación casi exclusiva de actores supranacionales, quienes han definido y conducido las políticas regionales.

En este orden, la integración económica constituyó una fuerte motivación a la hora de concertar espacios regionales, sin embargo, hoy se precisa de una integración que aborde problemáticas de mayor índole, estableciendo como prerrogativa escoger acciones y mecanismos en conjunto, a la par que emanar herméticamente un spill over holístico, es decir, un derrame que vaya más allá de las preferencias comerciales y se agolpe a la propia coyuntura social, política y cultural. En este sentido, tomando nuevamente las palabras de Haas (1970), los actores interpretarán positivamente cualquier tipo de interdependencia toda vez que sientan que los beneficios sean igualitarios. Estos actores son a priori los gobiernos centrales, pero, con el transcurso del tiempo, otros actores no estatales precisarán sostener su participación activa dentro de una gobernanza multinivel que sostenga la existencia de un nexo entre actores supranacionales y subnacionales y favorezca a la internacionalización de estos últimos (Botto, 2013).

Cabe mencionar que las múltiples organizaciones de la sociedad encuentran sus motivaciones considerando dos elementos interrelacionados: el grado de acceso al bloque y su capacidad en la toma de decisiones (Malamud, 2011). En lo que

1 Según Joseph Nye el poder duro implica la capacidad de usar palos (diplomacia coercitiva) o zanahorias (incentivos) para que otros acaten tu voluntad. 
respecta al MERCOSUR, esta situación se ve opacada por el carácter intergubernamental, que prácticamente inhibe a las unidades domésticas (Botto, 2013).

Con respecto a la complejidad de los instrumentos teóricos, es ideal tomar aquellas variables que revelan una interrelación directa del fundamento de este trabajo con sus correspondientes derivaciones, remarcando la actuación de los gobiernos locales en el sistema internacional y específicamente en la profundización de los PIR. Con el nacimiento de la Comunidad Europea del Carbón y el Acero (CECA), y posteriormente la firma del Tratado de Roma en el año 1957, se comenzaron a ensanchar distintas herramientas teóricas, intentando comprender un fenómeno que en siglos pasados ya fue desarrollando sus primeros pasos implícitamente. En este contexto, es oportuno valerse del neofuncionalismo, que, de acuerdo con uno de sus exponentes, Ernst Haas, supone un proceso dinámico y abierto, cuyo efecto derrame o spill over se proyectará inevitablemente en varias fracciones, más allá de la integración económica, alcanzando esferas sociales o políticas e incluso aquellas afines a la high politics, a través de la inmersión de una fortaleza institucional que garantiza la irreversibilidad de la integración. El efecto derrame, no obstante, no plasmó el desenlace previsto, lo que llevó a Haas a plantear el rol de los líderes políticos, sus objetivos, restricciones y motivaciones en medio de un conocimiento consensual (Malamud, 2011) que los lleva muchas veces a conformar un sistema decisorio en el avance de las negociaciones.

Posteriormente se sumaron los neofuncionalistas, como es el caso de Nye (1970), que subrayó variables de índole nacional y regional y sustentó los eventos que motivan los PIR, como la complementariedad de las elites, las transacciones y sus patrocinios, el rol de las presiones externas, los liderazgos y, aquí parte el punto más interesante en la elaboración de este artículo, la emergencia de actores no nacionales a pesar de que la integración regional se destaca por ser una decisión puramente estatal. El neofuncionalismo ayuda a comprender cómo los gobiernos no centrales se vinculan en un proceso de integración regional en medio de una multiplicidad de intereses (Oddone, 2016). En suma, los actores no nacionales desprenden una serie de mecanismos institucionales de carácter local que tienden a influenciar formalmente en los sistemas decisorios, sin embargo, dicha atribución se podría conjugar informalmente mediante la participación directa en sectores 
gubernamentales o privados y entes involucrados en los PIR (Passini y Pasquariello, 2002).

Otro instrumento significativo en la construcción de este artículo es el multilevel governance, que refuerza la capacidad y autonomía de gobiernos no centrales en los PIR y, con mayor acentuación, en la integración subnacional, que será ahondado en la próxima sección. Como afirman Granato y Oddone, governance comprende:

La capacidad o el proceso continuo mediante el cual diferentes intereses, muchas veces contrapuestos, logran ser reacomodados y constituirse en una estrategia cooperativa. Esto incluye la participación de instituciones formales y regímenes autorizados, así como los arreglos informales que los ciudadanos y las instituciones han convenido o perciben conveniente para la realización de un determinado interés (2010, p. 236).

Dentro de los límites de la gobernanza multinivel, prevalece la toma de decisiones compartidas en lugar de la monopolización por parte de gobiernos centrales, es decir, el funcionamiento de dichos Estados se complementa con la participación de actores a nivel subnacional (Huggins, 2015). En el contexto de la gobernanza multinivel y, de acuerdo con la analogía expresada por Peters y Pierre (2009), la UE es un modelo que no solo reconoce la existencia y la importancia de los gobiernos nacionales, sino que enfatiza la actuación de los gobiernos locales en la construcción de la política regional. En la misma línea, Sandholtz (1996) señala que las organizaciones internacionales u otro tipo de esquema regional con autonomía de poder, inevitablemente desarrollan un dominio de gobernanza multinivel en medio de una conjugación de dominios entre grupos domésticos y actores estatales.

Por su parte, Piattoni (2010) afirma que las organizaciones internacionales acaban influyendo en la movilización de las autoridades subnacionales y grupos sociales, instalando una mayor cooperación y coordinación a nivel internacional. Esta autora también puntualiza una creciente movilización y participación de dichos actores en la formulación de políticas a nivel subnacional y la prominencia del nivel territorial, que se interrelaciona con el interés de la clase política.

Cabe mencionar que el enfoque analítico de la gobernanza multinivel deja entrever la dispersión del poder en forma vertical u horizontal, desafiando el rol que cumplen los 
Estados desde diversas aristas y contribuyendo a reforzar la descentralización local (Granato y Oddone, 2010), lo que representa numerosos beneficios a los grupos subnacionales, quienes aprovechan sus ventajas comparativas para alistarse al sistema internacional, requiriendo y exigiendo su propia legitimación. A modo de ejemplificar este fenómeno, vale la pena tomar en cuenta la importancia que fue desarrollando el Comité Europeo de Regiones (CDR), un organismo consultivo de la UE que reconoció y promovió espacios de consultas y representación de las ciudades y regiones europeas, acentuando la cooperación descentralizada.

En adición, Huggins (2015) demuestra que la capacidad de los gobiernos no centrales y su vínculo a nivel supranacional conduce a la creación de "asociaciones transnacionales" entre actores subnacionales, o redes propiamente dichas. De hecho, puntualiza que las redes de gobiernos locales transnacionales (LGTN, por sus siglas en inglés) de la UE se enmarcan en el modelo de la gobernanza multinivel, con la particularidad de que esta gobernanza aglutina networkings en las cuales se explayan intereses comunes y compartidos, siendo uno de los grupos sociales más activos dentro de la integración europea. Participan activamente en el proceso político regional del bloque, a veces institucionalmente a través de la CDR o informalmente mediante lobbies en la ciudad de Bruselas. Sin embargo, el marco jurídico existente plantea un duro revés ante la falta de claridad de competencias, lo que lleva a considerar a los gobiernos locales como actores secundarios en la UE en el juego de la gobernanza multinivel. No solo redes locales pugnan por la participación de gobiernos locales en las políticas regionales, sino que también la academia y la sociedad civil impulsan estas iniciativas.

En el caso europeo podemos señalar Platforma, un hub líder en la internacionalización de gobiernos locales y regionales creada en 2008 y que desde sus antípodas trabaja arduamente como enlace de estas unidades subnacionales en la política supranacional de la UE (Platforma, 2020).

La UE ha abordado experiencias que permiten deducir que los gobiernos locales, en medio de una gobernanza multinivel, logran consolidar espacios relevantes en los PIR. A pesar de la falta de competencia en muchas áreas y la verticalidad impuesta en las relaciones con el gobierno central, los gobiernos locales han fortalecido su capacidad para la puesta en marcha de redes de cooperación e interacción, que 
conducen en un cierto plazo a una integración subnacional, un tipo de integración que merece una mayor focalización académica. Es el escenario donde las asimetrías estructurales y políticas no son de la envergadura de la integración supranacional y cuentan con una infraestructura flexible y menos burocrática, apegada a las demandas de sus propios habitantes, lo que permite en este nivel subnacional fomentar políticas locales efectivas en el marco de la integración.

La integración regional, desde la versatilidad que emanan sus principios, masifica impactos que inexorablemente recaen sobre actores subnacionales, y particularmente en los gobiernos locales. Ya sea en términos de cooperación o de nuevos enfoques transfronterizos, estas unidades colaboran en la construcción de una integración inclusiva, que exige a la supranacional una mirada desde abajo hacia arriba, que no reúne las condiciones del mainstream tradicional.

\subsection{La integración subnacional en el MERCOSUR}

El sistema internacional posterior al final de la Guerra Fría, junto con los impulsos de la globalización y las nuevas tecnologías, generó la aparición de múltiples actores dentro de los mismos Estados (gobiernos no centrales, grupos sociales, etc.) y fuera de ellos (organizaciones internacionales, entidades regionales, entre otros). Los asuntos internacionales como resultado de la interdependencia global exigen incluir en la agenda temas de dimensión humana tales como son los ambientales y sociales, cultura, infraestructura, educación o atención médica y de epidemias. La nueva agenda de las relaciones internacionales plantea asuntos que ahora son globales, pero de competencia local, que son "profunda e inseparablemente tanto internacionales como domésticos", lo que el autor acuña como asuntos intermésticos (Manning, 1977, p. 309).

En este panorama, en tendencia creciente y con fuerte dinamismo, aparecen actores subnacionales que cuestionan la exclusividad del Estado en las relaciones internacionales. Una de las primeras etiquetas que recibieron las actividades internacionales de actores subnacionales fue paradiplomacia -parallel diplomacy-. Esta diplomacia paralela nació conceptualmente en los años 80 y la mayor parte de la bibliografía refiere a Ivo Duchacek y Panayotis Soldatos como sus principales difusores en el ámbito académico (Duchacek, 1986; Soldatos, 1990). Cornago define la paradiplomacia como: 
La participación de gobiernos no centrales en las relaciones internacionales a través del establecimiento de contactos ad hoc con entidades privadas 0 públicas del extranjero, con el fin de promover asuntos socioeconómicos y culturales, así como cualquier otra dimensión externa de sus competencias constitucionales (2000, p. 66).

Camargo deja la paradiplomacia en manos de los gobiernos no centrales, es decir, los gobiernos intermedios y los gobiernos locales, y no de otro tipo de actores. Estos actores no nacionales pueden tener relaciones internacionales, pero no entrarían dentro del ámbito de la paradiplomacia. Nuestra posición converge con la vertida por Camargo, por lo que utilizaremos el término paradiplomacia únicamente para las unidades subnacionales o gobiernos no centrales.

Por gobiernos no centrales o subnacionales se entienden aquellas "unidades institucionales, o niveles del poder ejecutivo, componentes del gobierno de un Estado Nacional: estados, regiones, provincias, municipios u otros poderes locales" (Fronzaglia, 2005, p. 44). Vale aclarar que este trabajo se centra exclusivamente en los gobiernos locales (también llamados gobiernos municipales), instituciones a cargo de las funciones de administración local, que varía de acuerdo a las regulaciones legales y constitucionales de cada Estado o nación; asimismo se erige como el órgano subnacional de mayor relevancia en la ciudadanía, y esto se debe a tres aspectos básicos que lo enfatizan Destéfano, Lozano y Grisanti:

1. El núcleo de la administración municipal, que engloba las áreas centrales (administración financiera, rentas y catastro), incluyendo la organización y los procesos de trabajo.

2. La prestación de servicios públicos, de competencia históricamente municipal o más recientemente incorporados.

3. La generación de condiciones para el desarrollo integral, desde un enfoque de crecimiento equilibrado, inclusión social y sostenibilidad ambiental, que incluye la promoción del desarrollo local como uno de los roles actuales del gobierno municipal (2008, p. 1).

Los retos del siglo XXI son globales y no afectan exclusivamente a los Estados. Entre los principales desafíos globales encontramos el cambio climático, las migraciones 
internacionales, la revolución tecnológica y digital, las desigualdades en el interior de la sociedad y en el desarrollo de los países, la escasez de recursos, el empoderamiento ciudadano, la multipolaridad y las continuas demostraciones de fragilidad del multilateralismo. Paralelamente, el proceso de urbanización, cada vez más acelerado, alcanzó su apogeo en el año 2007 cuando la población urbana sobrepasó a la rural por primera vez en la historia ${ }^{2}$. Este hecho se vincula con el rol que juegan las grandes ciudades como centros de desarrollo económico y concentración de la población. Consecuentemente, las ciudades, desde la acción local, buscan gestionar estos problemas globales de los cuales reciben gran parte de sus consecuencias y ante los cuales los gobiernos nacionales reaccionan con demora. Al mismo tiempo, enfrentan problemas de tipo local que requieren soluciones globales. Por su parte, Cruz, Manassero y Piana (2019) incentivan la idea de "pensar globalmente, actuar localmente", cuya apertura parte de lo particular a lo universal, planteando una disyuntiva a los actores subnacionales en la actual sociedad internacional.

La integración regional o regionalismo abre las puertas a las primeras experiencias paradiplomáticas para la gran mayoría de los gobiernos no centrales gracias a los mayores flujos e interdependencias originados por la regionalización. Los PIR son procesos esencialmente supranacionales donde los Estados son los actores centrales y conducen y formalizan el proceso. Los fenómenos de la globalización, descentralización y la gobernanza multinivel permitieron a los gobiernos no centrales participar en estos procesos desarrollando proyectos de integración subnacional o microregionalismo (Malamud, 2011). Así es como comienzan a participar complementariamente, impulsando y sustentando la integración supranacional.

Desde este artículo consideramos como integración subnacional o microregionalismo a aquellos vínculos horizontales y en forma de red que se producen entre diferentes actores subnacionales en el marco de un proceso de integración supranacional. Estos pueden estar institucionalizados dentro de la estructura del bloque regional o producirse por fuera de ella ${ }^{3}$.

\footnotetext{
2 Para el año 2050 se estima que dos tercios de la población mundial vivirá en ciudades.

${ }^{3}$ Foro Consultivo de Ciudades y Regiones del MERCOSUR (FCCR) en el MERCOSUR o el Comité Europeo de las Regiones (CDR) en la UE.
} 
La integración latinoamericana es un proceso que se construye mediante comportamientos multinivel, asimismo el impacto per se de lo subnacional en lo regional transita constantemente en una dinámica de retroalimentación (Mellado, 2018). Los procesos de integración en América Latina se han centrado en el aspecto económico (conformación de un espacio de libre comercio), dejando de lado aspectos políticos, sociales y culturales. Como destaca Valle (2012), estas prácticas a nivel regional "ponen de manifiesto que los procesos de integración supranacional no han logrado generar beneficios sustanciales en las realidades nacionales" (p. 79). La creación de la ciudadanía regional y el rol del MERCOSUR para los ciudadanos descansa mayormente en los gobiernos locales. El municipio, como resalta Saavedra

Es el educador cívico por excelencia y a partir del cual es factible instalar en la base social una 'conciencia macro-regional' de modo que se convierta en el agente decodificador para el hombre común del para qué, del porqué y del para quién la integración regional del Cono Sur (1998, p.178).

Los gobiernos no centrales disponen de diferentes estrategias de relacionamiento internacional para vincularse. Las principales estrategias de participación en el sistema internacional son los hermanamientos, la integración transfronteriza, la constitución de redes de ciudades, la cooperación descentralizada y los distintos acuerdos bilaterales. Una modalidad emergente está apareciendo ante las nuevas necesidades de los gobiernos locales, lo que Pierre-Malé (2019) denomina "frentes" o "alianzas estratégicas de ciudades" 4

Duchacek (1990) clasifica las actividades de los gobiernos no centrales en tres categorías: paradiplomacia regional o fronteriza -los contactos se dan entre gobiernos subnacionales contiguos-, paradiplomacia transregional -los contactos se dan entre gobiernos subnacionales no contiguos pero cuyos Estados sí lo son- y paradiplomacia global -contactos directos con gobiernos subnacionales y gobiernos centrales en lugares distantes-. Las dos primeras modalidades se reproducen dentro de los bloques regionales, por lo que se puede afirmar que existe una relación intrínseca entre regionalismo y paradiplomacia (De Azevedo Banzatto, 2018).

${ }^{4}$ Es un espacio de presión directa e inmediata sobre los Gobiernos Nacionales para resolver problemas concretos o abolir determinadas políticas. 
En la región del MERCOSUR, estos vínculos horizontales y en red dentro del bloque regional, que tienen por objetivo promover y cohesionar la integración, se materializan a través de redes de ciudades de representación regional y la cooperación transfronteriza.

\subsubsection{Las redes de ciudades y Mercociudades}

Las redes de ciudades son organizaciones de gobiernos no centrales que se vinculan de forma horizontal (sin mando central jerarquizado) como instrumento que favorece la cooperación, desarrollo y consecución de objetivos. Estas se configuran como "espacios de aprendizaje, colaboración e intercambio donde confluyen el conocimiento y la experiencia práctica de los representantes políticos y técnicos de las ciudades" (AL-LAS, 2019, p. 27). Su rol protagónico en la acción internacional de los gobiernos locales se refleja en la proliferación que se ha producido desde los años 90 . Estudios recientes afirman que operan más de 200 redes multilaterales y foros donde se reúnen gobiernos locales (Acuto y Rayner, 2016). Estas redes tienen distintas formas y características, este trabajo se centra en aquellas que se crean con el objetivo de establecer un espacio de influencia y reconocimiento dentro de una estructura de instituciones supranacionales.

En el caso regional, en el año 1995 se funda Mercociudades, ${ }^{5}$ una red regional generalista de filiación pública y formada por los gobiernos locales de países pertenecientes al MERCOSUR y Sudamérica. Se conforma con el objetivo de aportar la dimensión local en los asuntos que les afectan en el seno del MERCOSUR y de cooperación entre sus miembros. Fundada por 11 ciudades, ha crecido continuamente y en la actualidad está compuesta por 351 ciudades (incluyendo ciudades de países que no forman parte del MERCOSUR), reflejando un fuerte interés en participar en la red. La creación de un foro que institucionalice la participación de las ciudades al más alto nivel en el proceso de integración ha sido una permanente reivindicación de las Mercociudades desde su nacimiento. Su actuación y perseverancia logró la creación en 2004 de un órgano permanente consultivo dentro de la estructura institucional del MERCOSUR: el Foro Consultivo de Municipios, Estados Federados, Provincias y Departamentos (FCCR), compuesto por

\footnotetext{
${ }^{5}$ Para un estudio sobre esta red, véase Alvaredo (2019).
} 
el Comité de Municipios (CM) y el Comité de Estados Federados, Provincias y Departamentos.

Mercociudades ha jugado un rol clave en aproximar a los gobiernos locales del MERCOSUR para compartir experiencias, elaborar proyectos en conjunto y construir una agenda compartida para enfrentar los desafíos y retos futuros (localizar los ODS, proyectos de cooperación sur-sur, construir la Nueva Agenda Urbana, etc.). Al mismo tiempo, ha logrado crear un espacio de legitimidad e influencia en el seno del MERCOSUR.

Sin embargo, la estructura propia del MERCOSUR fuertemente intergubernamental y la no obligación de tomar en consideración las propuestas de los órganos consultivos ha provocado una bajada constante de las reuniones celebradas hasta la nula celebración de reuniones en los años 2018 y 2019. Este proceso terminó de encallar en la Reunión LV/2019 del Consejo del Mercado Común, donde en la decisión 19/196 se realizó una actualización de la estructura institucional. En el artículo 8 se decide que el FCCR se constituirá como "órganos no permanentes que podrán celebrar reuniones bajo el formato de 'Conferencias', cuando juzguen necesario, para el tratamiento de temas de su competencia o cuando el GMC lo solicite". Mercociudades debe realizar una evaluación crítica del espacio para analizar si se apoya su continuidad o se apuesta a la construcción de un nuevo formato en la representación de "lo local" en el MERCOSUR.

A pesar de las dificultades que encuentran en su institucionalización, la conformación de redes de ciudades en los PIR, como es el caso de Mercociudades, fortalece a los gobiernos locales como actores con relevancia e implica a municipios próximos y distantes.

En este sentido, Chasquetti (2006) señala que la "experiencia de la Unión Europea muestra que la construcción de redes de gobiernos regionales y locales favorece la fortaleza del proceso de integración, brinda legitimidad a las decisiones, y agrega un actor en el desarrollo de políticas comunitarias" (p. 12). El autor destaca además cómo actualmente podemos observar en Europa "numerosas redes de ciudades y

${ }^{6}$ https://documentos.MERCOSUR.int/simfiles/normativas/75403 DEC 0192019 ES Actualizaci\%C3\%B3n\%20Estructura\%20Institucional.pdf 
regiones, con un importantísimo ámbito institucional de representación de la dimensión local: el Comité de Regiones, integrado por gobiernos regionales y municipales" (pp. 12-13)

\subsubsection{Cooperación transfronteriza}

Las fronteras son el espacio idóneo para la actuación internacional de los gobiernos no centrales. Es allí donde actúan diferentes niveles del Estado y se relacionan las comunidades fronterizas, dando lugar a la cooperación transfronteriza, entendida como la cooperación estratégica de los actores y territorios contiguos de Estados diferentes. Estudios demuestran que el hecho de pertenecer a un municipio de frontera aumenta las probabilidades de disponer de un área de relaciones internacionales en la estructura gubernamental, como el realizado por Matsumoto (2011), que identificó empíricamente que la posibilidad de que los municipios fronterizos de Brasil tengan un área internacional es 4.2 veces mayor que otros municipios brasileños no fronterizos. En el MERCOSUR es justamente en estas regiones donde la paradiplomacia se despliega en su mayor extensión, debido a que los gobiernos no centrales de las regiones fronterizas tienen mayor interés y necesidades en común, lo que facilita la cooperación.

Se han desarrollado diversas instancias institucionales para aproximarse a la temática fronteriza en el seno del MERCOSUR como el Grupo Ad Hoc de Integración Fronteriza (GAHIF), el Grupo de Trabajo de Integración Fronteriza (GTIF) del FCCR, la Comisión de Asuntos Fronterizos del Parlamento del MERCOSUR y el Fondo de Convergencia Estructural (FOCEM), destinado a proyectos de infraestructura y ya utilizado en proyectos de inclusión social en áreas fronterizas.

El GAHIF -creado en 2002- se reunió en pocas ocasiones y con escaso éxito en un formato donde no se convocaba a representantes de la población fronteriza ni de unidades subnacionales, sino que participaban los gobiernos nacionales con una lógica top down. EI FCCR, del cual ya se ha hecho mención en el apartado anterior, desde sus inicios tuvo como eje vertebrador la integración fronteriza. Se crea en su seno el Grupo de Trabajo de Integración Fronteriza que convoca provincias y municipios fronterizos. Tanto el FCCR como el GTIF fueron cayendo en sus actividades a pesar de distintos intentos de reactivación, como en el año 2015, en la XLV Reunión del FCCR, donde la presidencia pro tempore de Brasil propuso 
reactivar el GTIF y la creación de regiones de cooperación transfronteriza del MERCOSUR, las Mercoregiones. ${ }^{7}$ El "Acuerdo para la promoción de la integración fronteriza" propone espacios de acción conjunta referentes a mejorar los servicios públicos en estas zonas a través de consorcios públicos.

Durante el año 2019 se desarrollaron un número interesante de iniciativas en el MERCOSUR que hace pensar una creciente voluntad de estimular la temática fronteriza. Durante la XLVI reunión extraordinaria del Grupo Mercado Común, en febrero del año 2019, fue aprobada la creación del Subgrupo de Trabajo N. ${ }^{\circ} 18$ "Integración Fronteriza", que trabajará en articulación con espacios institucionales, referentes a temas de frontera, y tratará cuestiones relativas a salud, educación, trabajo, migración, transporte, infraestructura, desarrollo urbano, desarrollo económico, pueblos indígenas, cooperación, integración productiva y otras destinadas a impulsar la integración entre comunidades de frontera. Este nuevo subgrupo puede ser la oportunidad de reactivar algunas de las propuestas que quedaron postergadas por la falta de actividad del FCCR como las Mercoregiones.

Otra iniciativa de reciente creación y aprobada en diciembre del año 2019 en el LV Consejo del Mercado Común reunido en Bento Gonçalves, es el proyecto de "Acuerdo sobre localidades fronterizas vinculadas". El acuerdo busca facilitar la integración de las comunidades fronterizas e impulsar su integración con el objetivo de favorecer a los ciudadanos, promoviendo la cooperación en economía, tránsito, régimen laboral y acceso a servicios públicos. Se destaca la creación de un documento de tránsito vecinal fronterizo (DTVF), el acceso al régimen laboral y servicios públicos de salud, enseñanza y cultura, así como la cooperación en economía, sanidad pública, defensa civil y elaboración de planes de desarrollo urbano. Se encuentra pendiente de ser ratificado por parte de los países miembros del bloque. ${ }^{8}$

Paralelamente, instancias subnacionales también han desarrollado espacios extrainstitucionales a través de la formación de asociaciones interregionales y de

\footnotetext{
${ }^{7}$ http://portal.mercociudades.net/sites/portal.mercociudades.net/files/archivos/documentos/ ActasRemi/XLV reunion\%20de\%20coordinadores FCCR 15\%20de\%20julio 2015 Brasilia.p $\underline{\mathrm{df}}$

${ }^{8}$ https://www.mre.gov.py/tratados/public web/DetallesTratado.aspx?id=gGUe/CP6ZMa9ey Zri310sQ==\&em=Ic4aLYHVB0dF+kNrtEvsmZ96BovjLIz0mcrZruYPcn8=
} 
cooperación transfronteriza. Dentro de la red de Mercociudades las fronteras se trabajan en diversas unidades temáticas, pero la más activa es la de Integración Fronteriza, que trabaja de forma integral el asunto. Asimismo, se han constituido numerosas asociaciones subregionales con el propósito de facilitar la vinculación transfronteriza entre gobiernos subnacionales como son: la Asociación de Municipios de Fronteras Integradas del MERCOSUR -reúne localidades de Corrientes, Entre Ríos, sur brasileño y norte uruguayo-, el Comité para el Desarrollo de la Cuenca del Río Uruguay -nuclea a gobiernos locales de Argentina, Uruguay y Brasil-, CODESUL/CRECENEA Litoral -proyecto de integración subnacional que abarca los estados brasileños de Mato Grosso do Sul, Paraná, Santa Catarina, Rio Grande do Sul y las provincias del nordeste argentino de Corrientes, Chaco, Entre Ríos, Formosa, Misiones y Santa Fe-, ZICOSUR -foro de integración de carácter internacional integrado por provincias de Argentina, estados de Brasil, departamentos de Bolivia y Paraguay, regiones de Chile y Perú y Municipios de Uruguay-, Macro Región Atacalar -organismo de integración subnacional transfronteriza compuesto por las provincias argentinas de La Rioja, Catamarca, Tucumán, Santiago del Estero, Córdoba y Santa Fe y la región chilena de Atacamao el Bloque Regional de Intendentes, Prefeitos y Alcaldes del MERCOSUR (BRIPAM).

La cooperación transfronteriza es considerada la piedra angular en los procesos de integración. La temática fronteriza debe ser un eje del MERCOSUR y así es como ha ido desarrollando diferentes iniciativas institucionales, no obstante, sigue siendo necesario concretar un mayor número de mecanismos de consenso entre los distintos actores locales. Difícilmente se implementen medidas basadas exclusivamente en decisiones nacionales, sin permitir la participación de los gobiernos locales y las comunidades fronterizas. La participación de las unidades subnacionales, tal y como muestra la experiencia de la UE en la integración fronteriza, es fundamental para una efectiva y sostenible integración. Los espacios institucionales del MERCOSUR deben acompañar las iniciativas de carácter local que promuevan las relaciones transgubernamentales.

Es prioritario profundizar en la cooperación transfronteriza, ya que "las fronteras son un espacio territorial ideal para el desarrollo de diferentes esquemas de cooperación subnacional con miras a consolidar la integración regional" (Oddone, 2018, p. 75). 


\section{CONCLUSIONES}

Ante las complejidades que exhibe la coyuntura actual y, considerando el escenario pospandemia, la internacionalización de los gobiernos locales y el fortalecimiento de la integración regional son mandatos que engrosan el conjunto de respuestas esenciales ante el gran dilema que representaría la reconfiguración del sistema mundial. Este nuevo orden exigirá a priori una mayor participación de los actores subnacionales en la difícil travesía de recuperación, y a su vez en la implementación de políticas públicas y protección social, el intercambio de experiencias sanitarias, la reducción de asimetrías y desigualdades, la cooperación descentralizada, la provisión de bienes y servicios públicos de primera necesidad, el cumplimiento de la agenda 2030, el nuevo regionalismo y otras cuestiones prioritarias, que no son ajenas a los PIR.

La integración desde la perspectiva subnacional se erige como pilar trascendental en la profundización de los procesos de integración supranacional, y de esta forma permite avanzar hacia aquellas áreas desatendidas hoy por los gobiernos nacionales. La institucionalización de la integración a nivel local dentro del MERCOSUR se encuentra aún en un nivel embrionario, y al mismo tiempo afronta un conflicto de intereses frente a los gobiernos nacionales, cuando la realidad demuestra que es un eje notorio que promueve un plano de complementariedad, interdependencia y retroalimentación. La integración a nivel micro tiene una relación positiva con respecto a la integración supra provocando nuevos adeptos al esquema integracionista.

La respuesta a una ciudadanía más exigente, global y participativa, obliga a los gobiernos locales a buscar los mecanismos que respondan a las necesidades de sus habitantes, a pesar de no poseer atribuidas las competencias y el marco jurídico apropiado. Los municipios son la base de la vida democrática de la región -sus representantes son elegidos de forma directa- y cuentan con el privilegio de acceder a un mapa que refleja las realidades locales; además poseen la capacidad de promover una integración desde abajo -bottom up-, fomentando una gobernanza multinivel, de valioso aporte a los principios integracionistas. 
Se acercan los 30 años de nacimiento del MERCOSUR, y apostar por la integración subnacional podría convertirse en el camino ideal en tiempos que se discute la reformulación del bloque, y con ello sortear una integración regional fragmentada y fomentar una agenda más inclusiva. Se debe repensar este proceso de manera integral y evitar aquellos lineamientos volátiles que marcan el rumbo según sus agendas políticas. La actualidad parece indicar el regreso a una priorización del comercio con los países centrales, subordinado al resto de áreas irrelevantes muchas veces en el mainstream tradicional de la integración regional. El MERCOSUR debe abordar sistemáticamente la senda multidimensional, es decir, política, económica, social y cultural, a la par de consolidar un modelo de convivencia y cohesión regional, el cual se ajuste al objetivo prioritario del desarrollo. La integración subnacional puede ser una respuesta, ya que aceleraría la congruencia de la ciudadanía y la reducción de las asimetrías regionales transformándose en un prototipo dinamizador y reactivador del proceso.

MERCOSUR carece de espacios significativos que reconozcan la integración subnacional en su seno, ya que la propia estructura institucional e intergubernamental del bloque de algún modo los ha inhibido. Un apoyo al nivel de integración subnacional que reconozca institucionalmente a los gobiernos locales y sus procesos edificará una dimensión cercana a la realidad ciudadana. Es imperativo comprender que la conformación de espacios e iniciativas subnacionales en todos los ámbitos que confluyen dentro de la región descifra la clave hacia la legitimación del MERCOSUR que, luego de tres décadas de existencia, exige una reconfiguración que permita avanzar hacia una integración más profunda.

\section{BiBLIOGRAFÍA}

Acuto, M. y Rayner, S. (2016). City networks: breaking gridlocks or forging (new) lock$\begin{array}{llll}\text { ins? } & \text { International } & \text { 1147-1166. }\end{array}$ https://onlinelibrary.wiley.com/doi/full/10.1111/1468-2346.12700

Allas Proyecto. (2013). Marco Legal e Institucional para la acción internacional de las ciudades. Ciudad de México: Universidad Nacional Autonóma de México. 
AL-LAS. (2019). Alianzas locales para los retos globales. Cuadernos para la Internacionalización de las Ciudades, (9). https://proyectoallas.net/wpcontent/uploads/2019/10/cuadernoallas09 web-1.pdf

Alvaredo, N. (2019). Mercociudades: una red estratégica para Asunción y otros municipios del Paraguay. Instituto Social del MERCOSUR y Municipalidad de Asuncion.

Álvarez, M., Luna Pont, M. y Oddone, N. (2019). América Latina global: estudios regionales sobre paradiplomacia. Editorial de la Universidad Nacional de Tres de Febrero.

Botto, M. (2013). Integración regional y actores subnacionales. El caso del Mercosur. $\begin{array}{llll}\text { Temas } & y & \text { Debates, } & \text { 83-106. }\end{array}$ https://temasydebates.unr.edu.ar/index.php/tyd/issue/view/22

Congreso Nacional del Paraguay. Constitución Nacional. (1992). http://www.diputados.gov.py/ww5/index.php/legislacion/constitucion-nacional

Congreso Nacional del Paraguay. Ley №3966 Orgánica Municipal. (08 de febrero de 2010). https://www.bacn.gov.py/leyes-paraguayas/969/ley-n-3966-organica-municipal

Cornago, N. (2000). Diplomacia, Paradiplomacia y Redefinición de la seguridad mundial: Dimensiones de conflicto y cooperación. En F. Aldecoa y M. Keating (Eds.), Paradiplomacia: Las relaciones internacionales de las regiones (pp. 55-78). Marcial Pons.

Chasquetti, D. (2006). El MERCOSUR y las Ciudades. Apuntes para una agenda del Comité de Municipios del Foro Consultivo de Municipios, Estados Federados, Provincias, y Departamentos del MERCOSUR. Fundación Friedrich-Ebert-Stiftung. https://library.fes.de/pdf-files/bueros/uruguay/04478.pdf

Cruz, J., Manassero, M. y Piana, R. (2019). La política internacional subnacional: desafíos para los municipios de la Provincia de Buenos Aires en el siglo XXI. Revista Aportes para la Integración Latinoamericana, (39), 58-79. https://doi.org/10.24215/24689912e015 
de Azevedo Banzatto, A. P. (2018). A importância da paradiplomacia e da integração Fronteiriça para o fortalecimento da Integração regional no mercosul. En J. M. Ramos García y N. Oddone (Eds.), Integración y paradiplomacia transfronteriza: Experiencias comparadas del Río Bravo hasta la Patagonia (Tomo 2) (pp. 110-128). Colegio de la Frontera Norte.

Del Arenal Moyua, C. (2001). La nueva sociedad mundial y las nuevas realidades internacionales: Un reto para la teoría y para la política. Cursos de derecho internacional y relaciones internacionales de Vitoria-Gasteiz, 17-86. https://www.ehu.eus/documents/10067636/10664763/2001-Celestino-del-ArenalMoyua.pdf/bab55d27-64de-47f7-b3d3-0cf111044c18?t=1538566075000

Destéfano, E., Lozano Gracia, N. y Grisanti Bravo, J. (2018, 07 de agosto). Como fortalecer los gobiernos locales para beneficio de sus ciudadanos. Banco Mundial. https://blogs.worldbank.org/es/latinamerica/como-fortalecer-los-gobiernos-localespara-beneficio-de-sus-ciudadanos

Duchacek, I. (1986). The Territorial Dimension of Politics: Within, Among and Across Nations. Westview Press.

Duchacek, I., Latouche, D., y Stevenson, G. (1988). Perforated Sovereignties and International Relations: Trans-Sovereign Contacts of Subnational Governments. Greenwood.

Fronzaglia, M. (2005). Unidades Subnacionais e relações internacionais: um estudo de caso sobre a cidade de São Paulo - de 2001 a 2004 [Dissertação de Mestrado da Universidade Estadual de Campinas]. Repositório de UNICAMP, Brasil. http://www.repositorio.unicamp.br/handle/REPOSIP/279810

Granato, L. y Oddone, C. N. (2010). Entre las ciudades y los procesos de integración regional: Una apuesta a la governance multinivel. En M. A. Lopez y C. N. Oddone (Coords.), Las ciudades y los poderes locales en las relaciones internacionales contemporáneas (pp. 223-243). Unión Iberoamericana de Municipalistas.

Haas, E. (1958). The Uniting of Europe: Political, Social and Economic Forces 19501957. 
https://www.europarl.europa.eu/100books/en/detail/38/the-uniting-of-europe-politicalsocial-and-economical-forces-1950-1957

Haas, E. (1970). The Study of Regional Integration: Reflections on the Joy and Anguish of Pretheorizing. International Organization, 24(4), 606-646. https://doi.org/10.1017/S0020818300017495

Huggins, C. (2015). Local government transnational networking in Europe: a study of 14 local authorities in England and France [Doctoral Dissertation, Portsmouth University]. Portsmouth portal. https://researchportal.port.ac.uk/portal/en/theses/local-government-transnationalnetworking-in-europe(e90b229f-9a21-4dbb-986b-8240f8ffbcaa).html

Juste, S. (2016). Las unidades subestatales en el sistema internacional. Difusiones, 10(10), 38-56. https://www.revistadifusiones.net/index.php/difusiones/article/view/85

Keohane, R., y Nye, J. (1971). Transnational Relations and World Politics. Harvard University Press.

Keohane, R., y Nye, J. (1988). Poder e Interdependencia. La política mundial en transición. Grupo Editor Latinoamericano.

Malamud, A. (2011). Conceptos, teorías y debates sobre la integración regional. Norteamérica 6(2), 219-249. https://doi.org/10.22201/cisan.24487228e.2011.2

Malé, J. P. (2019). La emergencia de frentes y alianzas de ciudades: ¿Hacia nuevas formas de incidencia de los gobiernos locales? Monografías CIDOB, (72). https://www.cidob.org/es/content/download/72768/2341114/version/3/file/3340 JEAN-PIERRE\%20MAL\%C3\%89.pdf

Manning, B. (1977). The Congress, the Executive and Intermestic Affairs: Three $\begin{array}{llll}\text { Proposals. } & \text { Foreign 306-324. }\end{array}$ https://www.foreignaffairs.com/issues/1977/55/2

Marx, V. (2010). Las ciudades y su inserción política en las relaciones internacionales. En M. A. Lopez, y C. N. Oddone (Coords.), Las ciudades y los poderes locales en las relaciones contemporáneas (pp. 25-48). Unión Iberoamericana de Municipalistas. 
Matsumoto, C. E. H, (2011). As determinantes locais da paradiplomacia: o caso dos municípios brasileiros [Dissertação de Mestrado da Universidade de Brasília]. Repositório de UnB. https://repositorio.unb.br/handle/10482/10147

Mellado, N. (2018). MERCOSUR: de lo regional a lo subnacional. Revista Aportes para la Integración Latinoamericana, (39). https://doi.org/10.24215/24689912e012

Michelmann, H. J., y Soldatos, P. (1990). Federalism and International Relations: The Role of Subnational Units. Oxford University Press.

Nye, J. (1970). Comparing common markets: a revised neofunctionalist model. International Organization, 24(4), 796-835. https://doi.org/10.1017/S0020818300017537

Oddone, N., (2018a). Paradiplomacia transfronteriza: Reflexiones teóricas. En J. M. Ramos, y N. Oddone (Eds.), Integración y paradiplomacia transfronteriza: Experiencias comparadas desde el rio Bravo hasta la Patagonia (Tomo I, pp.49-100). El Colegio de la Frontera Norte.

Oddone, N. (2018b). ¿Y si probamos "resetear" el Mercosur desde las fronteras? La paradiplomacia transfronteriza y la nueva agenda del bloque regional. En J. M. Ramos García, y N. Oddone (Eds.), Integración y paradiplomacia transfronteriza: experiencias comparadas del Río Bravo hasta la Patagonia (Tomo II, pp. 89-108). EI Colegio de la Frontera Norte.

Oddone, N. (2016). La paradiplomacia desde cinco perspectivas: reflexiones teóricas para la construcción de una comunidad epistémica en América Latina. Relaciones Internacionales, 89(2), 47-82. http://dx.doi.org/10.15359/ri.89-2.2

Passini, M. y Pasquariello, K. I. (2002). As teorias de integração regional e os Estados subnacionais. Revista Impulso, 13(31), 47-69.

Peters, G., \& Pierre, J. (2009). Governance approaches. En A. Wiener y T. Diez, European Integration Theory (pp. 91-104). Oxford University Press.

Piattoni, S. (2010). The Theory of Multi-level Governance. Oxford University Press Platforma-dev.eu. (2020, 01 de Mayo). Quienes Somos. https://platformadev.eu/es/quienes-somos/ 
Rosales, O., Herreros, S., y Duran Lima, J. (2014). Integración regional: Hacia una estrategia de cadenas de valor inclusivas. Naciones Unidas CEPAL. https://www.cepal.org/es/publicaciones/36733-integracion-regional-estrategiacadenas-valor-inclusivas

Rosenau, J. (1990). Turbulence in World Politics: A theory of change and continuity. Princenton University Press.

Saavedra, O. (1998). Micromunicipios: entre el MERCOSUR y la descentralización. En O. Stahringer de Caramuti, EI MERCOSUR en el Siglo XXI. Ediciones Ciudad Argentina.

Snvdholtz, W. (1996). Membership Matters: Limits of the functional approach to European Institutions. Journal of Common Market Studies, 34(3), 403-429.

Valle Sosa, M. V. (2012). La emergencia de los actores locales en el plano internacional y los procesos subnacionales de integración regional: El caso de la ZICOSUR. Trabajos de Investigación en Paradiplomacia, (3), 75-86. https://issuu.com/equipoparadiplomacia/docs/tip3

Alvaredo, Nahuel Fernando. Universidad de Buenos Aires. Licenciado en Derecho y Administración de Empresas. Maestrando en Procesos de Integración Regional en la Universidad de Buenos Aires. alvaredo00@gmail.com

Arzamendia, Marcelo. Universidad de Buenos Aires. Magister en Procesos de Integración Regional con énfasis en el Mercosur. Especialista en Organización y Métodos, Licenciado en Administración de Empresas por la Universidad Nacional de Asunción. marceloarzamendiacuevas@gmail.com 